\title{
Zum Status eines mentalen Lexikons bei einem bilingualen Sprecher
}

ABSTRACT. On status of mental lexicon of bilingual people. Bilingual people have learnt the second language in a directed way, in institutionalised conditions, achieving linguistic and communicative competence close to competences of the first language, in reception as well as production. Do these people haev a common mental lexicon or two different ones? The author's research have shown that students posses two separate mental lexicons.

In diesem Artikel möchten wir uns mit dem bilingualen Lexikon beschäftigen, und genauer mit der Frage, ob sich bei den Zweisprachigen, die ihre zweite Sprache zwar im Kontext der ersten erlernt, jedoch sie in so einem Grade beherrscht haben, dass sie über eine sprachliche und kommunikative Kompetenz, die der der ersten Sprache gleicht, verfügen, ein gemeinsames oder zwei getrennte mentale Lexika herausgebildet haben.

In einer sehr allgemeinen Definition gilt das mentale Lexikon als derjenige Teil des Langzeitgedächtnisses, in dem die Wörter einer Sprache mental repräsentiert sind (Schwarz 1992: 81). Danach bildet es die Nahtstelle zwischen spezifischen Sinneseindrücken ${ }^{1}$ oder motorischen Mustern ${ }^{2}$ auf der einen Seite und mental repräsentierten Wissensstrukturen auf der anderen (vgl. Raupach 1997: 21). Der Begriff der mentalen Repräsentation verweist auf systeminterne Zustände, die systemexterne Zustände abbilden.

Die menschliche Sprache ist samt anderem Wissen im Gehirn gespeichert und in entsprechenden Situationen abrufbar. Markowitsch (1997: 27 f.) unterscheidet vier Gedächtnissysteme, von denen zwei deklarativer und weitere zwei nichtdeklarativer Natur seien. Bei den deklarativen Systemen ist das episodische bzw. autobiographische Gedächtnis für Ereignisse im

\footnotetext{
1 Bei der Sprachperzeption.

2 Bei der Sprachproduktion.
} 
Lebenslauf jedes Individuums zuständig, das Wissens- bzw. Faktensystem hingegen für Schul- oder Weltkenntnisse, aber auch für grammatische Regeln oder mathematische Formeln. Die zwei nichtdeklarativen Gedächtnissysteme differenziert er in ein prozedurales Gedächtnis, das erlernte und sodann weitgehend, automatisierte Bewegungsabläufe oder Handlungsstrategien umfasse, sowie die Prägung (priming), ein Wiedererkennen bzw. Erinnern von Reizen, Sinneseindrücken oder Situationen, die einem in dieser oder einer ähnlicher Weise früher bereits einmal begegnet sind. Diese Subkategorien des Gedächtnisses sind keineswegs voneinander getrennt, sondern gehen häufig ineinander über, was für den Sprachlernprozess von erheblicher Bedeutung ist. So wird der Fremdsprachenlernende sein explizit erlerntes Wissen im deklarativen Gedächtnis speichern, und mit der Zeit wird dieses Wissen automatisch angewendet. Zahlreiche Lernprozesse zielen daher darauf ab, deklaratives Wissen in prozedurales umzuwandeln (Götze 1999: 12).

An dieser Stelle soll auf den Unterschied zwischen den an sprachliche Einheiten geknüpften Bedeutungen auf der einen Seite und den sprachunabhängigen Konzepten als mentalen Grundeinheiten des menschlichen Wissens und Denkens auf der anderen Seite hingewiesen werden. Konzeptuelle Strukturen können prinzipiell unabhängig von den an sprachliche Formen gebundenen Repräsentationen existieren. Die kognitiven Konzepte beruhen auf der Organisation von Erfahrungsinhalten zu mentalen Einheiten und sind Grundeinheiten der mentalen Repräsentation. Die Konzepte haben eine individuelle Ausprägung. Die Einheiten der semantischen Organisation von Sprache sind dagegen überindividuell. Sie sind von ihrem Charakter her generalisierte und gesellschaftlich tradierte Kondensate kognitiver Konzepte. Ihre Funktion liegt in der Externalisierung individueller mentaler Repräsentationen (Möhle 1997: 43). Solche sprachlichen Bedeutungen gehören mit zu den Inhalten der Erfahrung von Realität, die den Aufbau des Systems kognitiver Konzepte beim Individuum bestimmen. Zwischen der mentalen Organisation von Erfahrungsinhalten und der sprachlichen Organisation von Wortinhalten besteht folglich eine Wechselwirkung. Daraus erklärt sich die beim Ablauf von Denk- und Sprechprozessen unauflösliche Verflochtenheit von kognitiven Konzepten und sprachlichen Bedeutungen.

Im Erstspracherwerb werden zunächst konzeptuelle Einheiten ausgebildet und im Langzeitgedächtnis repräsentiert, bevor sie mit phonologischen und morphosyntaktischen Einheiten verbunden und damit versprachlicht werden. Wird ihnen jedoch eine Wortform konventionellerweise zugeordnet, werden sie also lexikalisiert, so unterliegen sie nicht mehr nur den Erfordernissen der Kognition, sondern auch denen der Kommunikation und der grammatischen Strukturbildung. Das bedeutet, dass sie sozial akzeptiert und relativ stabil sein müssen und dass sie mit anderen lexikalisierten Beg- 
riffen strukturelle Beziehungen eingehen. Diese entsprechen denen, nach denen die Lexeme im Lexikon organisiert sind, also Relationen der semantischen Verbundenheit, bzw. des semantischen Kontrasts, der syntaktischen Kategorisierung, der morphologischen Zusammengehörigkeit und der phonologischen Ähnlichkeit. Konzepte sind also im Gedächtnis nicht isoliert abgespeichert, sondern sie sind durch verschiedene Relationen mit anderen Konzepten verknüpft. Die interkonzeptuellen Beziehungen stellen kognitive Strukturen dar, die Zusammenhänge eines Realitätsbereichs repräsentieren (Schwarz 1992: 87). Die lexikalische Bedeutung wird von uns als eine Funktion aufgefasst, die bestimmte konzeptuelle Schemata einer bestimmten Wortform zuordnet. Die Bedeutungen sind also von den reinen Konzepten zu unterscheiden und werden als Operationen auf konzeptuellen Schemata aufgefasst. Diese Operationen bestehen darin, der jeweiligen Wortform entsprechende konzeptuelle Schema zu suchen, zu aktivieren und gemäß dem aktuellen sprachlichen und nicht-sprachlichen Kontext zu konkretisieren, d.h. bestimmte Variablen durch dafür in Frage kommende Konștanten zu belegen (Scherfer 1997: 198).

Auch Erwachsene speichern Konzepte, ohne dass sie sprachverbunden sind, als mentale Repräsentationseinheiten und können sie notfalls in Paraphrasen oder Sätzen versprachlichen. Die zugrunde liegenden kognitiven Strukturprinzipien für die in den verschiedenen Sprachen vollzogenen Kategorisierungen sind universal und beziehen sich sowohl auf die Gliederung und Organisation des semantischen ${ }^{3}$ als auch des konzeptuellen ${ }^{4}$ Gedächtnisbesitzes. Diese Gleichartigkeit erklärt sich aus den biologisch-neurophysiologisch determinierten Grundpositionen menschlichen Wahrnehmungsund Denkverhaltens: Die vorsprachlichen konzeptuellen und verhaltensrelevanten Strukturbildungen behalten über den Prozess der Versprachlichung ihre grundlegende Gliederung und Organisation, auch in ihrer sprachlich fixierten Form, bei. Die Fähigkeit zu klassifizierender Wahrnehmung auf der Grundlage invarianter perzeptiver Merkmale, die Fähigkeit, verhaltensrelevante Objektklassen in hierarchisch organisierte Sequenzen zu strukturieren und mit Hilfe von Merkmalscharakteristiken im Gedächtnis zu fixieren sowie die Fähigkeit, an die Struktur von Objekten und Objekt- bzw. Verhaltenskonstellationen bestimmte Bedeutungen zu knüpfen, sind grundlegend für die kognitive Strukturbildungen und konstitutiv für alle Klassifikationsleistungen (Börner / Vogel 1997: 2f.). Das im Gedächtnis gespeicherte Wissen wird also als organisiertes Wissen aufgefasst und diese Organisation umfasst Repräsentationen (deklaratives Wissen) und kognitive Prozesse (prozeduralles Wissen). Die sprachlichen und außersprachlichen Informa-

\footnotetext{
${ }^{3}$ An sprachliche Formen gebundenen.

${ }^{4}$ Außersprachlichen, enzyklopädischen.
} 
tionen beruhen in Aufbau und Organisation auf denselben allgemein kognitiven Strukturprinzipien der klassifizierenden Wahrnehmung, der Kategorisierung, der Hierarchisierung und der Abstraktion. Lexikalische Einträge im mentalen Lexikon werden nicht nur auf einzelne holistische Konzepte abgebildet, sondern beziehen sich auch auf spezifische Merkmalskombinationen (Börner / Vogel 1997: 3f.). Das im mentalen Lexikon repräsentierte sprachliche Wissen besteht aus Formen, Inhalten und deren Strukturen, d. h. aus Einheiten sowie Operationen und ihnen zugrunde liegenden operativen Regeln, die die Bildung von Formen und Inhalten ermöglichen. Dabei stellen die Formen, Inhalte und Regeln komplex strukturierte Gebilde dar, die auf verschiedenen Strukturebenen mit jeweils unterschiedlichen Informationen organisiert werden (vgl. Miller 1978, Levelt 1989, Vogel 1990: 195):

- phonologische bzw. graphematische Information (Phonemstruktur, Silben und Akzentstruktur, Informationsmuster);

- morphologische Informationen (Flexionsmuster, Derivations- und Kompositionsschemata);

- syntaktische Informationen (Wortklassen, Valenz, Kasus);

- semantisch-lexikalische Informationen (Definition, Sinnrelationen, semantische Merkmale, Ähnlichkeit, Kontrast, Selektionsbeschränkungen);

- pragmatische Informationen (Situation, Rhetorik).

Zwischen den verschiedenen Einträgen des mentalen Lexikons bestehen sowohl intrinsische als auch assoziative Beziehungen (vgl. Möhle 1997: 46). Die sprachlichen Informationen werden also im mentalen Lexikon nicht isoliert, sondern in Form komplexer Zusammenhänge gespeichert. Wählt man versehentlich das falsche Wort, so wird dabei fast immer die Wortart des Zielwortes beibehalten - gleichgültig, ob es sich um eine Vertauschung auf Grund lautlicher oder semantischer Ähnlichkeit oder um eine Mischform aus beidem handelt. Demnach sind Nomen, Verben und Adjektive alle auf eine ganz bestimmte Weise strukturiert, was den Schluss nahe legt, dass Wortklassen im mentalen Lexikon mehr oder weniger eigene Wege gehen.

Wie wir schon erläutert haben, werden im mentalen Lexikon Informationen über einzelne Wörter gespeichert, die bei den Sprachproduktions- und Sprachverstehensprozessen abrufbar sind. $\mathrm{Zu}$ diesen Informationen zählt das unbewusste Sprachwissen über phonetisch-phonologische Formen, morphologische Strukturen, semantische Repräsentationen und syntaktische Regularitäten. Die Fähigkeit, sich relativ schnell aller Wörter zu bedienen, suggeriert, dass dieses Lexikon auf irgendwelche Weise geordnet, hierarchisiert und strukturiert sein muss. Die Wörter müssen im Kopf gut organisiert sein, weil sie buchstäblich in Sekundenbruchteilen abrufbar sind. Die meis- 
ten Menschen scheinen weniger als eine Sekunde zu brauchen, um ihren Wortspeicher gründlich zu durchsuchen, und sie finden beim Sprechen die benötigten Wörter beeindruckend schnell. Die große Anzahl von Wörtern, die ein Mensch kennt, und die Schnelligkeit, mit der er sie abrufen kann, deuten auf die Existenz eines perfekt organisierten mentalen Lexikons hin. Der menschliche Speicher ist anders als z. B. das Wörterbuch nicht nur nach Lautung oder Schreibung organisiert, wobei nicht nur der Anlaut, sondern auch andere Aspekte der Lautstruktur des Wortes, wie der Auslaut, das Akzentmuster, der Vokal mit dem Hauptakzent mitentscheidend sind. Auch die Bedeutung muss eine Rolle spielen, weil man recht häufig Wörter mit ähnlicher Bedeutung verwechselt. Wörter, die eng miteinander verwandt scheinen, sind in unmittelbarer Nähe voneinander gespeichert, oder sie sind voneinander entfernt, weisen aber eine starke Verbindung auf. Der Inhalt des mentalen Lexikons ist auch keineswegs begrenzt. Unablässig fügt man seinem Wortschatz neue Wörter hinzu und verändert Aussprache und Bedeutung existierender Wörter. Das wesentliche Strukturierungsprinzip der semantischen Informationen sind die Sinnrelationen, die zwischen den Lexemen bestehen können. Aitchison (1994: 84f.) verweist im mentalen Lexikon auf folgende semantische Beziehungen:

- Koordination - dieser Terminus steht für Wörter, die auf derselben Stufe der Hierarchie semantische Felder bilden. Diese sogenannten Konjunkte werden aber oft als Co-Hyponyme (benachbarte Unterbegriffe) bezeichnet, weil sie Hyponyme (Unterbegriffe) eines Hyperonyms (Oberbegriff) sind. Die Konjunkte stehen zueinander in der Relation der Inkompatibilität, d.h. sie schließen sich gegenseitig aus.

- Kollokation - bei dieser Erscheinung sind häufig auftretende Wortverbindungen, deren Miteinandervorkommen auf einer Regelhaftigkeit gegenseitiger Erwartbarkeit beruht (Bußman 1990: 391), im Gedächtnis in Form von semantischen Feldern abgespeichert. Hier herrscht die Relation der Kompatibilität vor. Aitchison zählt zu diesem Typ Wörter, die in zusammenhängender Rede oft mit dem Stimulus gemeinsam auftreten (kollokieren).

- Überordnung - die semantische Relation kennzeichnet die hierarchieähnliche Gliederung des Wortschatzes. Geeignete Oberbegriffe sind aber nicht immer leicht $\mathrm{zu}$ finden.

- Synonymie - die semantische Relation der Synonymie kennzeichnet die Bedeutungsgleichheit zwischen Wörtern. Häufig unterscheiden sich gleichbedeutende Wörter aber durch konnotative Merkmale und vor diesem Hintergrund sollte von Quasi-Synonymie gesprochen werden.

Einzelne Sprachen unterscheiden sich in ihrem Lexikon nicht nur dadurch, dass sie einzelne Konzepte unterschiedlich lexikalisieren, sondern 
vor allem auch dadurch, dass es nicht immer dieselben Konzepte oder Konzeptverknüpfungen sind, die lexikalisiert werden (vgl. Raupach, 1997: 26). Es ist also zwischen dem lexikalischen System einer Sprache, das die Menge aller möglichen lexikalischen Einheiten spezifiziert, und den Repräsentationen der kognitiven Konzepte, die sich sprecherindividuell aus jeweils subjektiven Erfahrungsinhalten entwickelt haben, zu trennen. Das konzeptuelle System ist ständigen Umstrukturierungen unterworfen. Außerdem muss hier angenommen werden, dass sich die Struktur des als prinzipiell sprachunabhängig modellierten konzeptuellen Systems eines Sprechers durch den Erwerb weiterer Sprachen ständig verändert.

Nach der bilingualistischen Theorie von Weinreich (1953: 9) verfügen die Zweisprachigen, die sich ihre zweite Sprache im Kontext der ersten angeeignet haben, über ein zusammengesetztes bzw. kombiniertes lexikalisches System, in dem Bedeutungsinhalte zweier Sprachen miteinander verschmelzen und lediglich formal unterschiedliche Ausdrücke auslösen. Das bedeutet, dass eine und dieselbe Bedeutung durch je ein Wort in beiden Sprachen ausgedrückt wird, d. h. der Zweisprachige verfügt über ein gemeinsames mentales Lexikon für beide Sprachen. Wir lassen Weinreichs Hypothese bezüglich des für beide Sprachen gemeinsamen lexikalischen Systems nur in der frühen Phase der Fremdspracherlernung zu. Am Anfang des Zweitspracherwerbsprozesses verfügen die Lernenden tatsächlich über ein für beide Sprachen gemeinsames mentales Lexikon, wobei das fremdsprachliche System mittels des muttersprachlichen erlernt wird (nach Weinreich haben wir hier mit der Subordination zu tun, d. h. eine Sprache hat den Zugang zum Bedeutungssystem über die andere). Interessant sind dabei nicht nur die im Gehirn gespeicherten Wörter, sondern auch der Weg, auf dem der lexikalische Zugriff nach allen mit diesen Wörtern verbundenen Informationen erfolgt. Bei einer nur geringen Beherrschung der Fremdsprache und bei einem Erlernen außerhalb von natürlichen Kommunikationssituationen wird eine Fremdsprache, genauer gesagt fremdsprachliche Bedeutungen von Wörtern, mit Hilfe muttersprachlicher Äquivalente gelernt. Im Gehirn wird kein neuer fremdsprachlicher Begriff ${ }^{5}$ strukturiert, es sei denn, es existiert noch keiner. Die kognitiven Strukturen, die sich im Prozess des Erstspracherwerbs in unserem Gehirn herausgebildet haben, werden also nicht aufs Neue gestaltet. Die Bedeutung eines fremdsprachlichen Wortes wird demnach auf der Basis des bekannten Konzeptes aufgebaut. Die Begriffe können mittels der Sprache geäußert werden, indem sie in eine graphische oder akustische Form gekleidet werden, der eine Bedeutung anhaftet. Im Falle der Erlernung einer anderen Sprache können sie höchstens modifiziert, differenziert oder neu miteinander verbunden werden. Wie wir schon ange-

${ }^{5}$ Die beiden Begriffe Begriff und Konzept betrachten wir als Synonyme. 
deutet haben, wird die Bedeutung als semantische Repräsentation der sprachlichen Einheit verstanden, mit der alle mit der Sprachstruktur verbundenen Informationen aktiviert werden. Damit der lexikalische Zugriff möglich ist, muss eine Verbindung zwischen der semantischen Repräsentation und dem Konzept bestehen.

Das folgende Modell schildert den Zugang zu Konzepten über die L1Bedeutungen zu Beginn des Zweitsprachaneignungsprozesses.

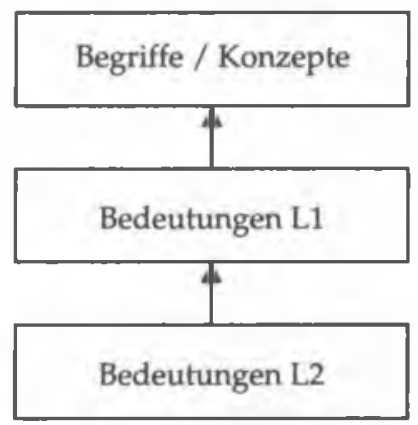

Über die erste Sprache wird also das Bedeutungssystem der zweiten Sprache aufgebaut und auf diese Weise bildet sich im Laufe der steigenden rezeptiven und produktiven Sprachkompetenz ein für beide Sprachen gemeinsames mentales Lexikon, in dem muttersprachliche Bedeutungen mit den erlernten fremdsprachlichen Äquivalenten verknüpft sind, und jede von diesen Bedeutungen mit anderen verwandten Bedeutungen entsprechender Sprache verbunden ist, d.h. einer L1-Bedeutung haftet ein L1-Synonym an und einer L2-Bedeutung haftet ein L2-Synonym an, die wiederum miteinander verknüpft sind. Abhängig davon, was man vom Lernenden fordert, benutzt er dementsprechend die Wortformen der Mutter- oder der Fremdsprache. Man kann dies an einem Modell veranschaulichen.

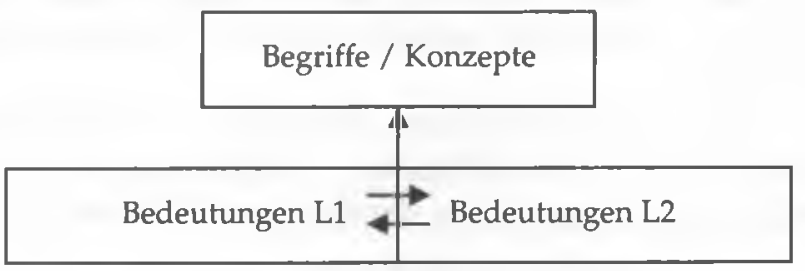

In diesem Stadium des Erlernungsprozesses existieren die L2-Bedeutungen nicht in sich selbst, sondern in fester Verbindung mit den L1-Bedeutungen. Während der kognitiven Verarbeitungsprozesse des fremdsprachlichen Input oder Output werden gleichzeitig muttersprachliche lexikalische Einheiten aktiviert. 
Nach ungefähr sechs / sieben Jahren des intensiven Studierens ${ }^{6}$ trennt sich dieses lexikalische System, d.h. die fremdsprachliche Bedeutung wird nicht mehr über oder mit der muttersprachlichen aktiviert. Es bilden sich zwischen den Konzepten und den eigenständigen Bedeutungssystemen der L1 und L2 Netzverbindungen heraus, so dass der lexikalische Zugriff nach fremdsprachlicher Einheit direkt erfolgen kann.

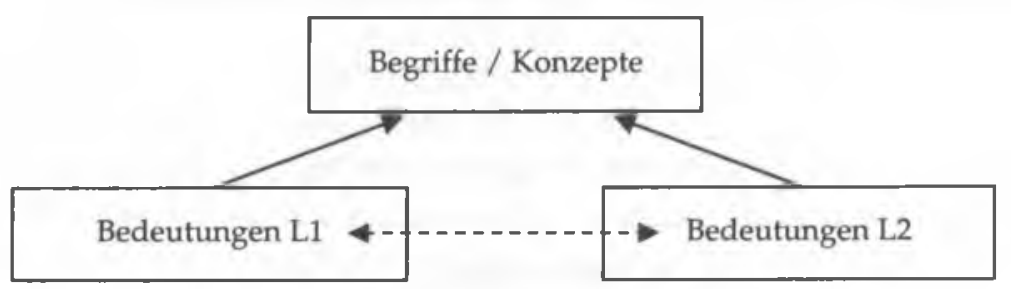

Für den Lernenden beginnen die fremdsprachigen Bedeutungen als ungebundene Teile eines mentalen Lexikons zu existieren, d. h. er kann die in der Fremdsprache verfassten Sätze bzw. Texte ohne den Bezug auf die Muttersprache kodieren, dekodieren und weiterverarbeiten. Die L2-Bedeutungen werden nicht mehr mit den L1-Bedeutungen, sondern mit anderen L2-Einheiten innerhalb des entsprechenden Lexikons assoziiert. Die Bilingualen, bei denen sich zwei getrennte lexikalische Systeme herausgebildet haben, verfügen weiterhin über ein gemeinsames Begriffssystem, wo das Weltwissen in Form von Konzepten gespeichert wird. Die Bedeutungssysteme sind dagegen stark mit der jeweiligen Sprache verbunden und haben direkten, d. h. ohne Vermittlung der anderen Sprache, Zugang zu diesen Begriffen. Die geschilderten kognitiven Vorgänge sind nur im Falle eines sehr großen Grades an Fortgeschrittenheit im Fremdsprachenlernprozess, der eigentlich schon abgeschlossen wurde, weil sich im Gehirn bereits alle nützliche fremdsprachlichen Strukturen herausgebildet haben, möglich. In einer solchen Situation haben wir es mit einem vollständigen ${ }^{7}$ und balancierten Bilingualismus zu tun und verzichten auf die unzutreffende Bezeichnung von Weinreich 8 .

Um unsere These zu unterstützen, haben wir zwei Jahrgänge der Germanistikstudenten des 3. Studienjahres - Repräsentanten der noch nicht vollständigen Zweisprachigkeit, bei denen aber schon die Erscheinung des

${ }^{6}$ Wir meinen hier nicht den gewöhnlichen Unterricht in der Schule, wo die L2 wie andere Fächer gelehrt wird, sondern die Umstände, wo die L2 nicht nur zum Gegenstand des Unterrichts, sondern auch zum Mittel der Kommunikation und des Wissensgewinnens wird und damit den Rang der L1 erreicht.

${ }^{7}$ Es ist hier ein sehr hoher Grad der sprachlichen und kommunikativen Kompetenz gemeint.

${ }^{8}$ Der zusammengesetzte Bilingualismus. 
Trennungsprozesses eines gemeinsamen Bedeutungssystems in zwei den beiden Sprachen entsprechende Systeme zu beobachten ist, untersucht (Legutko-Marszałek 2003). Die Probanden haben Polnisch als die erste Sprache erworben und dann in der Schule mit dem Lernen der zweiten Sprache (Deutsch) begonnen. L2 wurde also erlernt, d.h. bewusst angeeignet und nicht wie L1 auf natürlichem Wege erworben. Die Analyse des mentalen Lexikons wurde mittels der Methode der gerichteten (kontrollierten) Assoziationen (vgl. Kurcz 2000: 110) durchgeführt. Die Studenten bekamen eine Liste mit den gewisse Assoziationen hervorrufenden Wörtern in deutscher (L2) oder in polnischer (L1) Sprache und ihre Aufgabe bestand darauf, mit der verbalen Reaktion in entsprechender Sprache auf den Primärreiz zu antworten. Die sprachliche Reaktion wurde in dem Sinne kontrolliert, dass die Untersuchten angewiesen wurden, mit einem Antonym, Synonym oder einem Wortfeld zu agieren. Die Tests wurden in einem vier- oder dreimonatigen Abstand durchgeführt, um zu vermeiden, dass sich die Studenten die angegebenen Wortbedeutungen in der einen Sprache merkten, die dann die Assoziationen in der anderen beeinflussen könnten. Jeder Student bekam also dieselbe Aufgabe, aber in einer jeweils anderen Sprache.

Bei der Überprüfung der Ergebnisse haben wir berücksichtigt, wie viele Antonyme bzw. Synonyme in polnischer und in deutscher Sprache im Vergleich zu den nicht ausgefüllten Stellen eingesetzt worden waren. Von den ausgefüllten Assoziationen waren all die von besonders großer Bedeutung, die sich in der polnischen und der deutschen Fassung unterschieden. Voraussetzung für den Vergleich der eingesetzten Synonyme bzw. Antonyme und für die Absonderung der einstimmig und unterschiedlich ausgefüllten Wörter war die Tatsache, dass die Wörter in den beiden Sprachen, d. h. sowohl in der deutschen als auch in der polnischen eingetragen wurden. In anderem Fall blieben die Assoziationen unberücksichtigt. Unberücksichtigt blieben auch die falsch verstandenen und unleserlich eingesetzten Wörter. Wir haben angenommen, dass im Falle der Übereinstimmung der Assoziationen in den beiden Sprachen die Untersuchten über ein gemeinsames mentales Lexikon für L1 und L2 verfügen. Bei Nicht-Übereinstimmung jedoch erlaubten wir uns anzunehmen, dass sich bei ihnen bereits zwei getrennte lexikalische Systeme herausgebildet haben, die beiden Sprachen entsprechen.

Wir konnten auf Grund der Ergebnisse der durchgeführten Experimente beobachten, dass die Assoziationen in Form der Synonyme und Antonyme in deutscher und in polnischer Sprache in großem Maße nicht übereinstimmten (Legutko-Marszałek 2003: 106-159, 2003: 163-248). Sogar bei Ausfüllung der Wortfelder, wo die Studenten die Möglichkeit hatten, mehrere Assoziationen einzutragen und die Wahrscheinlichkeit der Übereinstimmung erheblich war, konnte man große Unstimmigkeiten beobachten. Obwohl unsere Hypothese der getrennten mentalen Lexika bei analysierter Art 
des Bilingualismus die Absolventen der Germanistik betrifft, die sich einer hervorragenden Sprachkompetenz erfreuen, wird schon in der durchgeführten Untersuchung sichtbar, wo der Prozess des Fremdspracherlernens noch nicht abgeschlossen ist, dass die Probanden nicht über ein für die beiden angeeigneten Sprachen gemeinsames Bedeutungssystem verfügen können, wie es Weinreich behauptete. Daraus lässt sich schlussfolgern, dass zwei sprachspezifische Bedeutungssysteme nicht nur auf den koordinierten Bilingualismus zutreffen und dass die Zweisprachigen, die ihre Fremdsprache in dem Sprachkontext der ersten Sprache gelernt haben, nicht unbedingt über ein gemeinsames Lexikon verfügen müssen, in dem die Bedeutungen der beiden erlernten Sprachen paarweise anhaften und bei der Aktivierung einer Bedeutung in der deutschen Sprache gleich der polnische Äquivalent mitaktiviert wird. Die untersuchten Studenten verbanden manchmal völlig andere Assoziationen mit dem angebotenen Reizwort in der deutschen und völlig andere in der polnischen Sprache. Die Assoziationsunterschiede betrafen Sprachebene, Wortart, Konkretheit vs. Abstraktheit, ganz andere Vorstellungen bezüglich der Bedeutung in beiden Sprachen usw. Das erlaubt uns anzunehmen, dass die Bedeutungen einer Sprache stark miteinander vernetzt sind und nicht mit denen der anderen Sprache verschmelzen und lediglich formal unterschiedliche Ausdrücke auslösen.

Das mentale Lexikon der einen Sprache muss irgendwie anders organisiert sein als das der anderen, d.h. je nach den Regularitäten des lexikalischen Systems einer entsprechenden Sprache. Man könnte auch vermuten, dass die Probanden größere Probleme mit der Ausfüllung der Assoziationen in der Fremdsprache haben sollten, weil sie noch nicht vollständig erlernt worden ist, was bei unserer Untersuchung nicht der Fall war. Wie aus der Analyse der Ergebnisse (Legutko-Marszałek 2003: 102f., 2003: 162f.) hervorgeht, war die Zahl der unterschiedlich ausgefüllten Assoziationen im Vergleich zu der Zahl aller ausgefüllten Assoziationen verhältnismäßig groß. Das bestätigt unsere Annahme, dass bei den Studenten des 3. Jahres der Prozess der Lexikontrennung bereits eingetreten und bei besonders fortgeschrittenen Studenten fast abgeschlossen ist. Natürlich gab es auch einstimmig ausgefüllte Assoziationen, aber das ist keinesfalls auf die Zusammengesetztheit der L1- und L2-Bedeutungen zurückzuführen, sondern eher mit der Spezifik der Reizwörter verbunden. Es gab nämlich Wörter, die keine Alternative zuließen, weil sie so stark mit der außersprachlichen Welt zusammenhängen, dass sie beinahe in jeder Sprache assoziativ verbunden sind, wie z.B. kalt-warm, schwarz-wei $\beta$, krank-gesund, klug-dumm usw.

Auf Grund der von uns durchgeführten Experimente vertreten wir die Meinung, dass sich bei den Zweisprachigen, die über eine hervorragende sprachliche und kommunikative Kompetenz verfügen, egal, ob sie die zweite Sprache im Kontext der ersten erlernt oder in einem anderen, nur für die 
zweite Sprache reservierten Kontext erworben haben, im Laufe der Zweitsprachaneignung zwei getrennte und unabhängige Bedeutungssysteme herausgebildet haben. Die Bilingualen, die eine Fremdsprache zwar in der Schule erlernt, aber nahezu perfekt beherrscht haben, können nicht über ein zusammengesetztes Lexikon verfügen, weil:

- die Bedeutungsumfänge der zwei verschiedenen Sprachen sich sehr oft nicht überlappen;

- eine zweisprachige Person eine fremdsprachliche Bedeutung genauso schnell aktivieren kann wie eine einsprachige, d.h. der lexikalische Zugriff nach einer sprachlichen Einheit nimmt genauso viel Zeit in Anspruch, obwohl sie doppelt so viel Einheiten gespeichert hat;

- bei Zweisprachigen die kognitiven Verarbeitungsprozesse in jeder der beiden Sprachen belanglos verlaufen und völlig automatisiert sind;

- die Bilingualen in beiden Sprachen ohne Störungen kommunizieren können und in jedem Moment von einer in die andere umschalten können;

- die Zweisprachigen versprechen, d.h. das falsche Wort aktivieren, nur in der Sprache, in der sie momentan sprechen;

- die L1-Bedeutungen die L2-Bedeutungen und umgekehrt nicht voraktivieren;

- die kontrollierten Assoziationen in Form von Synonymen und Antonymen nicht übereinstimmen.

Verschiedene Kontroversen erweckt auch die Frage, ob die Beherrschung von zwei Sprachen die Organisationsstruktur des Gehirns verändert. Manche Untersuchungen haben ergeben, dass die linke Hemisphäre des Gehirns bei der Verarbeitung sowohl der ersten als auch der zweiten Sprache dominiert. Andere wiesen wiederum auf die verminderte Überlegenheit der linken Gehirnhälfte hin und sogar auf die Einschaltung der rechten Hemisphäre (vgl. List 1995: 28). Letztens wurde ein Experiment mit Hilfe der PETTechnik $^{9}$ durchgeführt, das direkt dieses Problem betraf (Klein / Milner / Zatorre / Meyer / Evans 1995a, b). Ziel der Untersuchung war es, die Kortexrepräsentationen für Sprache bei den Bilingualen zu finden. Die Ergebnisse der Positronenemissions-Tomographie haben ergeben, dass der größte Blutdurchfluss während allen Aufgaben in der linken Hirnhälfte zu beobachten war, unabhängig davon, in welcher Sprache die Aufgaben gelöst wurden. In keinem Fall wurde die Aktivierung der rechten Hemisphäre bestätigt.

In den neuesten Experimenten, die die Repräsentationen der Sprachfunktionen bei den Bilingualen betrafen, wurde die fMRI-Methode ${ }^{10}$ ange-

9 Positronenemissions-Tomographie.

${ }^{10}$ Die funktionelle Kernspinresonanz-Tomographie. 
wendet (Kim / Relkin / Lee / Hirsch 1997). Die Probanden sollten in Gedanken die Sätze formulieren, die die Ereignisse aus dem vorigen Tag beschreiben sollten. Vor jedem Gehirn-Scanning wurden sie instruiert, welcher Sprache sie sich bedienen sollten. Auf Grund der erbrachten Ergebnisse kamen die Forscher zu einer Feststellung, dass in dem die Sprachfunktionen kontrollierten Gehirnareal die zweite Sprache, die nach der Pubertätsphase erlernt wurde, räumlich von der ersten abgeschottet ist. Die Sprachen aktivierten nämlich andere Hirnstellen, je nachdem, welche im Moment benutzt wurde. Diese Untersuchungen bestätigen auch unsere These, dass die Zweisprachigen, die ihre zweite Sprache nach der ersten im institutionalisierten Unterricht erlernt haben und sie in solchem Maße beherrscht haben, dass sie rezeptiv und produktiv in jeder Situation entsprechend sprachlich agieren können, über die nach Sprachen getrennten mentalen Lexika verfügen müssen.

\section{BIBLIOGRAPHIE}

Aitchison, J., 1994. Words in the mind. An introduction to the mental lexicon. Oxford UK and Cambridge USA: Blackwell (second edition).

Albert, R., 1998. Das bilinguale mentale Lexikon. In: Deutsch als Fremdsprache 35, 90-97.

Börner, W. / Vogel, K., 1997. Mentales Lexikon und Lemersprache. In: Börner, W. / Vogel, K. (Hg.): Kognitive Linguistik und Fremdsprachenerwerb. Tübingen: Gunter Narr Verlag.

Felix, S., 1982. Psycholinguistische Aspekte des Zweitsprachenerwerbs. Tübingen: Narr.

Friederici, A.D., 1994. Gehirn und Sprache. Neurobiologische Grundlagen. In: Krämer, S. (Hg.): Geist - Gehirn - Künstliche Intelligenz. Zeitgenössische Modelle des Denkens. Berlin: Walter de Gruyter Verlag: 113-130.

Götze, L., 1999. Der Zweisprachenerwerb aus der Sicht der Hirnforschung. In: Deutsch als Fremdsprache 36: 10-16.

Kim, K. / Relkin, N.R. / Lee, K. / Hirsch, J., 1997. Distinct Cortical Areas with Native and Second Languages. In: Nature, Bd. 388: 171-174.

Klein, D. / Milner, B. / Zatorre, R.J. / Meyer, E. / Evans, A.C., 1995a. The Neutral Substrates Underlying Word Generation: A Bilingual Funktional-Imaging Study. In: Proceedings of the National Academy of Sciences USA, Bd. 92: 2899-2903.

Klein, D. / Milner, B. / Zatorre, R.J. / Meyer, E. / Evans, A.C. (1995b): The Neutral Substrates of Bilingual Language Processing: Evidence from Positron Emission Tomography. In: Paradis (Hg.): Aspects of Bilingual Aphasia. London: Pergamon.

Kurcz, I., 2000. Psychologia języka i komunikacji. Warszawa: Wydawnictwo Naukowe Scholar.

Legutko-Marszałek, I., 2003. Lexikalische Systeme als bilinguale Wirklichkeit. Uniwersytet Gdański: Wydzial Filologiczno-Historyczny (nicht publizierte Doktorarbeit).

Levelt, W.J., 1989. Speaking: From Intention to Artikulation. Cambridge. Mass.: Brandford \& MIT Press.

List, G., 1995. Zwei Sprachen und ein Gehirn. Befunde aus der Neuropsychologie und Überlegungen zum Zweitspracherwerb. In: Fremdsprache Deutsch - Sondenummer.

Markowitsch, H.J., 1997. Neuropsychologie des menschlichen Gedächtnisses. In: Spektrum der Wissenschft 4: 27-28.

Miller, G., 1978. Semantic relations among words. In: Halle, M. / Bresnan, J. / Miller, G. (des.): Linguistic Theory and Psychological Reality. Cambiridge (Mass.): M.I.T. Press: 60-118. 
Möhle, D., 1997. Deklaratives und prozedurales Wissen in der Repräsentation des mentalen Lexikons. In: Börner, W. / Vogel, K. (Hg.): Kognitive Linguistik und Fremdsprachenerwerb. Tübingen: Gunter Narr Verlag.

Raupach, M., 1997. Das mehrsprachige mentale Lexikon. In: Börner, W. / Vogel, K. (Hg.): Kognitive Linguistik und Fremdsprachenerwerb. Tübingen: Gunter Narr Verlag.

Scherfer, P., 1997. Überlegungen zu einer Theorie des Vokabellernens und -lehrens. In: Börner, W. / Vogel, K. (Hg.): Kognitive Linguistik und Fremdsprachenerwerb. Tübingen: Gunter Narr Verlag.

Schwarz, M., 1992. Einführung in die Kognitive Linguistik. Tübingen: A. Francke Verlag. Schwarze, Ch. / Wunderlich, D., 1985. Handbuch der Lexikologie. Königstein: Athenäum.

Vogel, K., 1990. Lernersprache. Linguistische und psycholinguistische Grundfragen zu ihrer Erforschung. Tübingen: Gunter Narr Verlag.

Weinreich, U., 1953. Languages in Contact: Findings and Problems. In: Publications of the Linguistic Center of New York 1. New York. 